Internist 2019 $60: 1136-1145$

https://doi.org/10.1007/s00108-019-00671-5

Online publiziert: 27. August 2019

(c) Springer Medizin Verlag GmbH, ein Teil von Springer Nature 2019

\section{Redaktion}

C.F. Vogelmeier, Marburg

T. Welte, Hannover

\author{
V. M. Corman' ${ }^{1}$ J. Lienau ${ }^{2} \cdot$ M. Witzenrath ${ }^{2,3}$ \\ ${ }^{\prime}$ Institut für Virologie, Charité - Universitätsmedizin Berlin, corporate member of Freie Universität Berlin, \\ Humboldt-Universität zu Berlin, and Berlin Institute of Health, Berlin, Deutschland \\ ${ }^{2}$ Arbeitsbereich Pulmonale Inflammation, Charité - Universitätsmedizin Berlin, corporate member of Freie \\ Universität Berlin, Humboldt-Universität zu Berlin, and Berlin Institute of Health, Berlin, Deutschland \\ ${ }^{3}$ Medizinische Klinik mit Schwerpunkt Infektiologie und Pneumologie, Charité - Universitätsmedizin \\ Berlin, corporate member of Freie Universität Berlin, Humboldt-Universität zu Berlin, and Berlin Institute \\ of Health, Berlin, Deutschland
}

\title{
Coronaviren als Ursache respiratorischer Infektionen
}

nur ein kleiner Teil der bekannten $\mathrm{CoV}$ humanpathogen (•Tab. 1). Bei symptomatischen Infektionen lösen $\mathrm{CoV}$ im Regelfall respiratorische Erkrankungen aus. Vier der bisher bekannten sechs humanpathogenen $\mathrm{CoV}$ werden weltweit im Menschen gefunden und sind je nach Patientenkollektiv für $10-15 \%$ der akuten respiratorischen Erkrankungen (ARE) verantwortlich [10, 15, 24]. Zusätzlich zu diesen ständig im Menschen zirkulierenden Varianten wurden in den vergangenen Jahren zwei CoV im Menschen gefunden, nämlich SARS-CoV und MERS-CoV, die aus dem Tierreservoir auf den Menschen übergegangen sind und bei einem deutlich größeren Anteil der Infizierten schwere virale Pneumonien mit tödlichem Verlauf auslösen [25, 28].

\section{\) Bei symptomatischen Infektionen lösen CoV im Regelfall respiratorische Erkrankungen aus} terfamilie Orthocoronavirinae (Ordnung Nidovirales, Familie Coronaviridae) klassifiziert. Die Unterfamilie Orthocoronavirinae umfasst eine große Anzahl an verschiedenen Genera, Subgenera und Virusspezies [12]. Darunter sind viele Varianten, die in der Veterinärmedizin sowohl für Haustiere (etwa bezüglich der felinen infektiösen Peritonitis bei Katzen) als auch für Nutztiere (schwere Diarrhöen bei Rindern und Schweinen) von Bedeutung sind.

Bisher wurden sechs verschiedene $\mathrm{CoV}$ in Menschen gefunden, folglich ist
[28]. Die meisten Infektionen traten bisher in Ländern der Arabischen Halbinsel auf. MERS-CoV zirkuliert seit mindestens 2012 kontinuierlich in dieser geografischen Region, sodass eine Infektion mit MERS-CoV bei passender Anamnese auch in Europa differenzialdiagnostisch relevant ist.

In diesem Beitrag geben wir einen kurzen Überblick über alle sechs im Menschen vorkommenden $\mathrm{CoV}$ mit Fokus auf das hochpathogene und aktuell noch zirkulierende MERS-CoV.

\section{Die vier in Menschen weltweit endemischen Coronaviren}

Mit HCoV-OC43 und -229E wurden zwei der vier endemischen humanpathogenen $\mathrm{CoV}(\mathrm{HCoV})$ bereits in den 1960 er-Jahren beschrieben. Die zwei weiteren endemischen $\mathrm{CoV}$ (HCoV-NL63 und-HKU1) wurden erst deutlich später, in den 2000er-Jahren entdeckt [5]. Alle vier $\mathrm{CoV}$ sind etablierte humanpathogene Erreger, die weltweit vorkommen und klassischerweise ARE auslösen können. Je nach Patientenkollektiv werden Hinweise auf eine aktive CoV-Infektion bei bis zu $20 \%$ der Patienten mit akuten ambulant erworbenen respiratorischen Erkrankungen gefunden, sowohl bei Kindern als auch bei Erwachsenen [10, 15, 24]. Asymptomatische Infektionen wurden ebenfalls beschrieben [23].

Obwohl die Mehrzahl der Infektionen mit den vier endemischen CoV nur leichte Atemwegserkrankungen verursacht, können alle $\mathrm{HCoV}$ auch schwere 
Hier steht eine Anzeige.

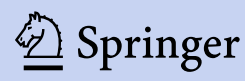


Tab. 1 Übersicht über die im Menschen vorkommenden Coronaviren

\begin{tabular}{|c|c|c|c|c|}
\hline Virus & $\begin{array}{l}\text { Bekannt } \\
\text { seit }\end{array}$ & Typische Klinik & Infektionsquelle für Menschen & Schwerpunkt der geografischen Verbreitung \\
\hline $\mathrm{HCoV}-\mathrm{OC} 43$ & 1960 & ARE, ILI & Mensch & Weltweit \\
\hline HCoV-229E & 1960 & ARE, ILI & Mensch & Weltweit \\
\hline HCoV-NL63 & 2003 & ARE, ILI & Mensch & Weltweit \\
\hline HCoV-HKU1 & 2004 & ARE, ILI & Mensch & Weltweit \\
\hline SARS-CoV & 2004 & Virale Pneumonie & Fledermaus, Schleichkatzen, Mensch & Seit 2004 keine menschlichen Fälle mehr (vorher Ostasien) \\
\hline MERS-CoV & 2012 & Virale Pneumonie & Dromedare, Mensch & Arabische Halbinsel \\
\hline
\end{tabular}

Krankheitsverläufe induzieren. Dies betrifft insbesondere Risikogruppen, wie immunsupprimierte Patienten, Patienten mit pulmonaler Vorerkrankung und Säuglinge, selten aber auch Patienten ohne spezifisches Risikoprofil [8].

\section{Die beiden zoonotischen Coronaviren}

Zusätzlich zu den vier endemischen $\mathrm{HCoV}$ haben in den letzten zwei Jahrzehnten die beiden epidemischen $\mathrm{CoV}$ SARS-CoV und MERS-CoV beim Menschen schwere Atemwegserkrankungen ausgelöst.

\section{SARS-Coronaviren}

SARS-CoV war der verursachende Erreger eines Ausbruchs viraler Pneumonien in den Jahren 2002/2003. Mindestens 8000 Menschen wurden im Rahmen des Ausbruchs durch SARS-CoV infiziert und etwa $10 \%$ starben infolge der Infektion. SARS-CoV ist ein zoonotisches Virus, das sein Tierreservoir in bestimmten Fledermausarten hat. Es wird angenommen, dass für den Übergang zum Menschen auch Wildkatzen, die für den menschlichen Verzehr gehalten wurden, eine Rolle gespielt haben. Der weitere Verlauf des SARS-CoV-Ausbruchs war dann durch Mensch-zu-Mensch-Infektionsketten geprägt [5]. Der Ausbruch hatte seinen geografischen Ursprung in China und der Großteil der menschlichen Infektionen war ebenfalls in Ostasien zu beobachten. Durch Reisetätigkeit infizierter Personen waren aber mehr als 25 Länder weltweit betroffen (• Abb. 1a; [25]).
Da seit Anfang 2004 keine Fälle von humanen SARS-CoV-Infektionen mehr detektiert wurden, kann davon ausgegangen werden, dass SARS-CoV aktuell nicht mehr im Menschen vorkommt. Da SARS-CoV im Tierreservoir weiterhin zirkuliert, sind jedoch Mensch-zuMensch-Übertragungsketten nach einem erneuten Übergang von SARS-CoV aus Tieren auf den Menschen möglich. Solch ein erneuter Übergang ist jedoch als eher unwahrscheinlich einzuschätzen, da eine deutlich erhöhte Aufmerksamkeit hinsichtlich Infektionserregern in Fledermäusen und Wildkatzen besteht, insbesondere im Zusammenhang mit dem Handel für den menschlichen Konsum.

\section{MERS-Coronaviren}

MERS-CoV ist das zweite hochpathogene CoV, das im Menschen gefunden wird. Es wurde bei Menschen erstmalig 2012 im Verlauf einer fatalen viralen Pneumonie in Saudi-Arabien entdeckt [11, 14, 28, 30]. Seitdem wurden etwa 2500 humane MERS-CoV-Infektionen gemeldet, wovon etwa $30 \%$ tödlich verliefen.

Über $90 \%$ der Infektionen traten in Ländern der Arabischen Halbinsel auf, besonders in Saudi-Arabien. Importierte Fälle wurden aber auch in europäischen Ländern einschließlich Deutschland dokumentiert, insgesamt sind bisher mehr als 25 Länder betroffen (• Abb. 1b; [28]). In manchen Ländern kam es ausgehend von einem importierten Fall auch zu weiteren autochthonen Infektionen. Besonders zu erwähnen ist hierbei der MERSCoV-Ausbruch in Südkorea im Jahr 2015, bei dem ausgehend von einem einzelnen infizierten Reiserückkehrer mehr als 160 weitere nosokomiale Infektionen resultierten, unter anderem auch von medizinischem Personal [11].

Neben Mensch-zu-Mensch-Übertragungen im häuslichen Umfeld und im Krankenhaus stellen Dromedare auf der Arabischen Halbinsel die wichtigste Infektionsquelle dar $[11,14]$. MERS-CoV zirkuliert seit mindestens 2012 kontinuierlich in dieser geografischen Region, sodass eine Infektion mit MERS-CoV bei passender Anamnese weltweit differenzialdiagnostisch relevant ist [26].

Interessanterweise stellen Dromedare auch außerhalb der Arabischen Halbinsel ein Reservoir für MERS-CoV dar. So wurde das Virus auch in Dromedaren in West- und Ostafrika sowie in Asien nachgewiesen (- Abb. 1b und 2; [2, 13, 31]). Primäre zoonotische Infektionen wurden aus diesen Regionen allerdings noch nicht bekannt.

\section{Einordnung als „priority diseases"}

Seit 2015 stehen die beiden pandemischen CoV (SARS- und MERS-CoV) auf der von der Weltgesundheitsorganisation (WHO) aufgesetzten Liste der „priority diseases“. Diese Liste verzeichnet die acht Infektionskrankheiten, bei denen zukünftig eine weltweite Epidemie für möglich gehalten wird und für die ein dringender Forschungs- und Entwicklungsbedarf zur Verhinderung einer Ausbreitung gesehen wird [17]. 
Internist 2019·60:1136-1145 https://doi.org/10.1007/s00108-019-00671-5

(c) Springer Medizin Verlag GmbH, ein Teil von Springer Nature 2019

\section{M. Corman $\cdot$ J. Lienau $\cdot$ M. Witzenrath}

\section{Coronaviren als Ursache respiratorischer Infektionen}

\section{Zusammenfassung}

Hintergrund. Es gibt sechs humanpathogene Coronaviren $(\mathrm{CoV})$, die vornehmlich respiratorische Infektionen auslösen. Im klinischen Alltag ist es hilfreich, Relevanz und Besonderheiten dieser Erreger zu kennen. Fragestellung. Praxisnahe Darstellung der Epidemiologie, Klinik und Unterschiede humanpathogener CoV. Hinweise zu Diagnostik und Umgang mit Patienten bei Verdacht auf eine CoV-Infektion.

Material und Methoden. Selektive Literaturrecherche, Darstellung und Diskussion von Grundlagenarbeiten und Expertenempfehlungen einschließlich der Veröffentlichungen der Weltgesundheitsorganisation (WHO), des Europäischen Zentrums für die Prävention und die Kontrolle von Krankheiten (ECDC) und des Robert Koch-Instituts.
Ergebnisse. Die weltweit endemischen CoV (HCoV-NL63, -229E, -OC43 und -HKU1) sind häufige Ursache von leichteren respiratorischen Infektionen. Zwei epidemische, zoonotische Coronaviren (SARS- und MERSCoV, MERS "Middle East respiratory syndrome", SARS "severe acute respiratory syndrome" [schweres akutes Atemwegssyndrom]) können schwere Pneumonien hervorrufen. SARS-CoV ist seit 15 Jahren nicht mehr im Menschen nachgewiesen worden. MERSCoV zirkuliert seit 2012 hauptsächlich auf der Arabischen Halbinsel. Eine spezifische Therapie oder zugelassene Impfung existiert noch für keines der sechs humanpathogenen Coronaviren.

Schlussfolgerung. Eine Erregerdiagnostik mittels Polymerase-Kettenreaktion aus respiratorischem Material ist für alle $\mathrm{CoV}$ möglich, für die vier endemischen allerdings selten notwendig. SARS-CoV hat aktuell keine Relevanz in der Klinik, da es seit 15 Jahren nicht mehr im Menschen nachgewiesen wurde. Der Verdacht auf eine MERS-Infektion sollte hingegen bei entsprechender Symptomatik und Reiseanamnese differenzialdiagnostisch in Betracht gezogen werden. Hier sind eine schnelle Diagnosesicherung und allgemeine Hygienemaßnahmen zur Verhinderung der Verbreitung entscheidend.

\section{Schlüsselwörter}

Pneumonie - Akute respiratorische Erkrankungen - Middle-East-respiratorysyndrome-Coronavirus - Schweres akutes Atemwegssyndrom · Erkältung

\section{Coronaviruses as the cause of respiratory infections}

\section{Abstract}

Background. There are six human pathogenic coronaviruses (CoV), which mainly cause infections of the respiratory system. In everyday clinical practice, it is helpful to know the relevance and characteristics of these pathogens.

Objective. To present the epidemiology, clinical picture and differences of human pathogenic $\mathrm{CoV}$ and to provide information on the diagnostics and treatment of patients suspected of having CoV infections.

Material and methods. Selective literature search, presentation of results and discussion of fundamental works and expert recommendations, including publications by the World Health Organization (WHO), the European Centre for Disease Prevention and Control (ECDC) and the Robert Koch Institute.
Results. The four endemic human CoVs (HCoV-NL63, HCoV-229E, HCoV-OC43 and HCoV-HKU1) mainly cause mild respiratory tract infections. In addition to these four endemic $\mathrm{HCoV}$, the two epidemic $\mathrm{CoV}$, severe acute respiratory syndrome (SARS)-CoV and Middle East respiratory syndrome (MERS)-CoV can cause severe pneumonia. The SARS-CoV has not been detected in humans in the last 15 years and MERS-CoV has been circulating mainly on the Arabian Peninsula since 2012; however, neither a specific treatment nor approved vaccines exist for any of the six human pathogenic CoVs.

Conclusion. All six human CoVs can be diagnosed using RT-PCR on respiratory specimens but this is rarely necessary for the four endemic strains. In current clinical practice SARS-CoV has no importance as it has not been detected in humans for 15 years; however, a possible MERS-CoV infection should be taken into account in patients with typical symptoms and travel history to endemic regions. In this case, rapid diagnostic and general hygiene practices are important to prevent further transmission.

\section{Keywords}

Pneumonia - Respiratory tract infections . Middle East respiratory syndrome coronavirus (MERS-CoV) - Severe acute respiratory syndrome (SARS) · Common cold

\section{Klinische Symptome}

\section{Endemische Coronaviren}

Die vier endemischen und weltweit häufigen $\mathrm{CoV}$ verursachen in der Regel nach 2-10 Tagen Inkubationszeit leichte bis mittelschwere Infektionen der oberen Atemwege mit den typischen Symptomen einer Erkältung bzw. eines grippalen Infekts, das heißt mit Schnupfen, Husten,
Halsschmerzen, Kopfschmerzen, subfebriler oder febriler Temperatur und allgemein reduziertem Wohlbefinden.

Nahezu alle Menschen werden im Laufe ihres Lebens mit diesen CoVVarianten infiziert. Aufgrund einer nur temporären Immunität sind auch Reinfektionen mit demselben Typ häufig. Neben dem typischen klinischen Bild einer ARE können die endemischen $\mathrm{CoV}$ in seltenen Fällen auch schwere
Erkrankungen der unteren Atemwege wie Pneumonie oder Bronchitis verursachen. Dies ist häufiger der Fall bei Menschen mit kardiopulmonalen oder malignen Vorerkrankungen, unter Immunsuppression sowie bei Säuglingen und älteren Erwachsenen. 


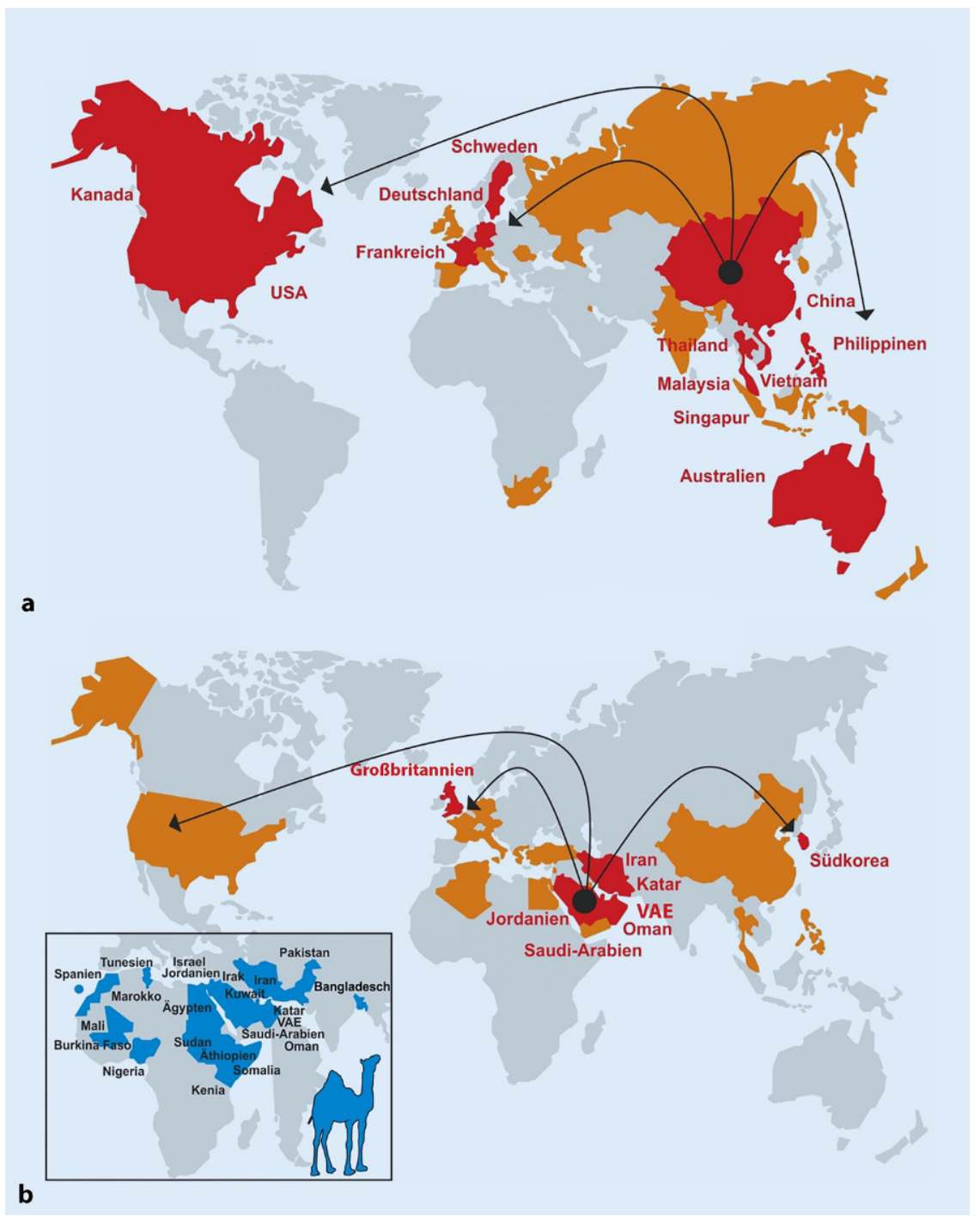

Abb. 1 a a SARS-CoV (2002/2003); b MERS-CoV (2012 bis heute). Rot dargestellt und bezeichnet sind alle Länder mit mindestens 5 SARS- bzw. MERS-CoV-Fällen; orange die Länder mit weniger als 5 Fällen; blau alle Länder mit Nachweis von MERS-CoV in Dromedaren. CoV Coronavirus, MERS „Middle East respiratory syndrome“, SARS "severe acute respiratory syndrome" (schweres akutes Atemwegssyndrom), VAE Vereinigte Arabische Emirate

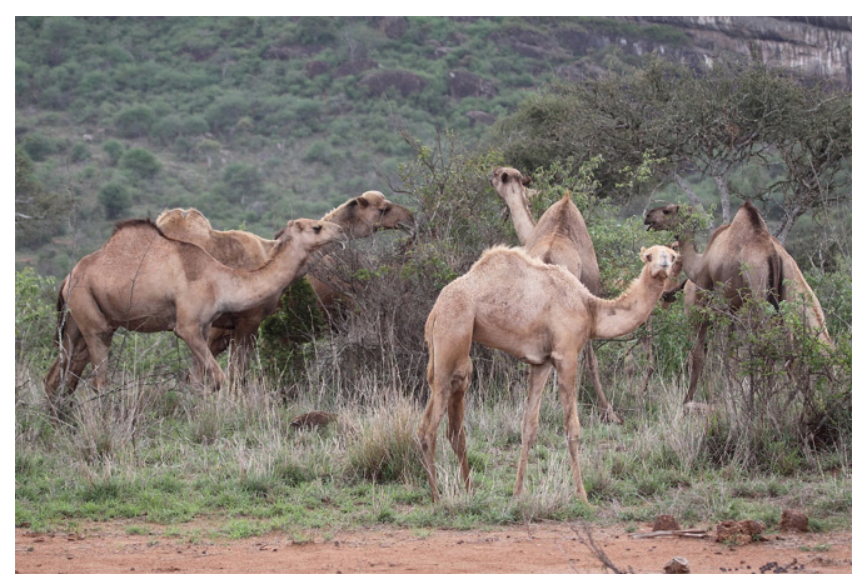

Abb. $2<$ Einhöckrige Kamele (Camelus dromedarius) stellen das tierische Reservoir für MiddleEast-respiratory-syndrome-Coronaviren (MERS-CoV) dar. (Mit freundl. Genehmigung $\odot$ V.M. Corman, alle Rechte vorbehalten)

\section{MERS-Coronaviren}

Ähnlich wie bei den häufigen endemischen $\mathrm{CoV}$ beträgt die Inkubationszeit der MERS-CoV-Infektion 2-14 Tage. Das klinische Spektrum einer MERSCoV-Infektion reicht ebenfalls von milden Symptomen oder leichten Atemwegsbeschwerden bis hin $\mathrm{zu}$ schweren akuten Atemwegserkrankungen und Tod. Durchschnittlich verläuft die MERS-CoV-Infektion jedoch gravierender als die endemischen CoV-Infektionen und imponiert typischerweise als Pneumonie mit Fieber, Husten und Atemnot bis hin zum akuten Lungenversagen. Auch bei Fällen ohne Fieber und Husten, bei denen basierend auf klinischen oder radiologischen Befunden der Verdacht auf eine Beteiligung der unteren Atemwege besteht, sollte bei entsprechender Anamnese an eine Infektion mit MERS-CoV gedacht werden. Die Auswertung der bisherigen Fälle deutet darauf hin, dass MERS-CoV vor allem bei älteren Menschen, immunsupprimierten Patienten und Patienten mit chronischen Erkrankungen der Niere oder der Lunge sowie bei Patienten mit Diabetes oder einem Tumorleiden schwere Erkrankungen verursacht. Allerdings ist durch den hohen Anteil nosokomialer Fälle eine genauere Analyse bezüglich Vorerkrankungen als Risikofaktor schwierig.

\section{》) Die MERS-CoV-Infektion imponiert typischerweise als Pneumonie mit Fieber, Husten und Atemnot}

Ein Aufenthalt als Patient oder als medizinischer Mitarbeiter in einer Klinik in der von MERS-CoV betroffenen Region kann grundsätzlich ebenfalls als Risikofaktor betrachtet werden. Für ambulant erworbene MERS-CoV Infektionen scheint neben dem Kontakt zu MERS-CoV-Infizierten vor allem ein enger regelmäßiger Kontakt zu Kamelen der wichtigste Risikofaktor zu sein. Etwa 35\% der bisher gemeldeten Patienten mit bestätigter MERS-CoVInfektion sind im Laufe der Infektion 
gestorben $[11,28]$. Da leicht verlaufende Erkrankungen häufig nicht gemeldet werden, ist die Sterblichkeitsrate aber wahrscheinlich deutlich geringer.

\section{SARS-Coronaviren}

Die Symptome einer SARS-CoV-Infektion sind vergleichbar mit den Symptomen einer MERS-CoV-Infektion. Da in den letzten 15 Jahren keine menschlichen SARS-CoV-Infektionen mehr bekannt wurden, verzichtet diese kurze Übersichtsarbeit auf eine detaillierte Betrachtung der SARS-CoV-Infektionen.

\section{Koinfektionen}

Virale Koinfektionen der Lunge, unter anderem mit Influenza A und B, Parainfluenza, Rhinovirus und Adenovirus, sind sowohl bei der Infektion mit endemischen als auch bei Infektionen mit MERS- und SARS-CoV beschrieben worden. Koinfektionen mit Bakterien kommen auch gelegentlich vor. Bei einigen Patienten mit SARS/MERSCoV-Infektion wurde zusätzlich von sekundär nosokomial erworbenen nichtviralen Infektionen (Pneumokokken, Staphylokokken, Klebsiellen, Acinetobacter, Candida) berichtet. Inwiefern diese sekundären Infektionen auf die intensivmedizinische Behandlung und Beatmung zurückzuführen sind oder ein spezifisches grundsätzliches Risiko einer CoV-Infektion darstellen, ist noch nicht verstanden.

Neben dem regelhaften Nachweis von $\mathrm{CoV}$ im Respirationstrakt sind alle endemischen CoV auch in Stuhlproben nachgewiesen worden; sie sind jedoch typischerweise keine kausale Ursache einer Gastroenteritis [9, 20]. Zudem gibt es Hinweise, dass Infektionen mit $\mathrm{HCoV}$ OC43 eine Rolle bei neurologischen Erkrankungen spielen könnten [18, 29].

\section{Diagnostik}

Die Unterscheidung zwischen $\mathrm{CoV}$ und anderen Erregern, die eine ARE auslösen, ist anhand der klinischen Symptome grundsätzlich nicht möglich. Jedoch ist eine spezifische Labordiagnostik bei Verdacht auf eine Infektion mit endemi- schen CoV bei harmlosem Verlauf und Patienten ohne besonderes Risiko für die Entstehung von Komplikationen auch nicht indiziert. Bei komplizierten Verläufen und schweren Infektionen des Respirationstrakts ist eine Testung auf $\mathrm{CoV}$ mittels Echtzeit-Reverse-TranskriptasePolymerase-Kettenreaktion (RT-PCR) möglich und sinnvoll. Geeignetes Probenmaterial sind Abstriche oder Sekrete aus dem oberen, je nach Klinik auch aus dem unteren Respirationstrakt. Eine Diagnostik über den Nachweis von Antikörpern ist aufgrund der häufigen (Re-)Infektionen nur in Ausnahmefällen sinnvoll und begründet.

\section{MERS-Coronaviren}

Eine spezifische Untersuchung auf eine Erkrankung durch MERS-CoV muss beim Vorliegen bestimmter Kriterien durchgeführt werden. Mittel der Wahl ist auch für MERS-CoV der Direktnachweis der viralen RNA mittels RT-PCR [3, 14, 26, 27]. Geeignete Materialien sind vor allem Sekrete aus den unteren Atemwegen (bronchoalveoläre Lavage, Absaugsekrete, Sputum), da dort die höchsten Viruskonzentrationen vorliegen [4]. Ist die Gewinnung und Testung dieser Materialien nicht möglich, können auch Abstriche aus dem oberen Respirationstrakt (Nasen/Rachen) untersucht werden.

\section{》) Die Quantifizierung spezifischer Antikörpertiter ist für die Abklärung einer MERS-CoV- Infektion ungeeignet}

Die Antikörperdiagnostik basierend auf der Testung von zwei konsekutiven Serumproben, beispielsweise im Abstand von 4 Wochen, ist für den Ausschluss einer akuten Infektion ungeeignet und nur in bestimmten Situationen (Umgebungsuntersuchung, Postexpositionsuntersuchung) indiziert [26]. Aufgrund der interindividuellen Heterogenität der Antikörperantwort bei MERS-CoVInfektionen ist die Quantifizierung spezifischer Antikörpertiter zum Nachweis oder Ausschluss einer MERS-CoV-In- fektion ungeeignet. In - Abb. 3 ist dargestellt, wann und wie in einem MERS$\mathrm{CoV}$-Verdachtsfall vorgegangen werden sollte.

Bei der typischerweise unspezifischen Klinik von MERS-CoV-Infektionen sollte auch die Möglichkeit einer Infektion mit anderen Pathogenen in Betracht gezogen werden [26]. Bei Patienten, für die laut MERS-CoV-Falldefinition eine Diagnostik indiziert war und von denen Proben zum Ausschluss einer MERS-CoVInfektion an das Konsiliarlabor für $\mathrm{CoV}$ gesendet wurden, werden regelmäßig andere Erreger als MERS-CoV als ursächlich für die respiratorische Erkrankung identifiziert. Häufig handelt es sich dabei um (eigene unpublizierte Daten)

- Influenzavirus,

- endemisches CoV,

- Rhinovirus,

- Enterovirus,

- Parainfluenzavirus,

- respiratorisches Synzytialvirus,

- humanes Metapneumovirus und

- Adenovirus.

Auch an typische und atypische Erreger einer bakteriellen Pneumonie wie Mykoplasmen, Chlamydien oder Legionellen sollte differenzialdiagnostisch gedacht werden.

Aktuelle Informationen für das Vorgehen bei Verdacht auf eine Infektion mit MERS-CoV werden auf der Website des Robert Koch-Instituts (RKI) zur Verfügung gestellt (https://www.rki.de).

\section{Übertragung}

Die Mensch-zu-Mensch-Übertragung der endemischen $\mathrm{CoV}$ erfolgt durch Tröpfchen, die unter anderem beim Niesen entstehen und über geringe Distanz $(<10 \mathrm{~m})$ auf die Haut und von dort auf die Schleimhäute anderer Personen gelangen können, wo es dann zu Infektionen von Schleimhautzellen kommt. Auch eine aerogene Übertragung durch sehr kleine Tröpfchen, die länger in der Luft schweben oder über weitere Strecken transportiert werden können, wird diskutiert. Zusätzlich spielt die direkte Übertragung durch mit virushaltigen Sekreten kontaminierte Hände und Oberflächen eine Rolle. 


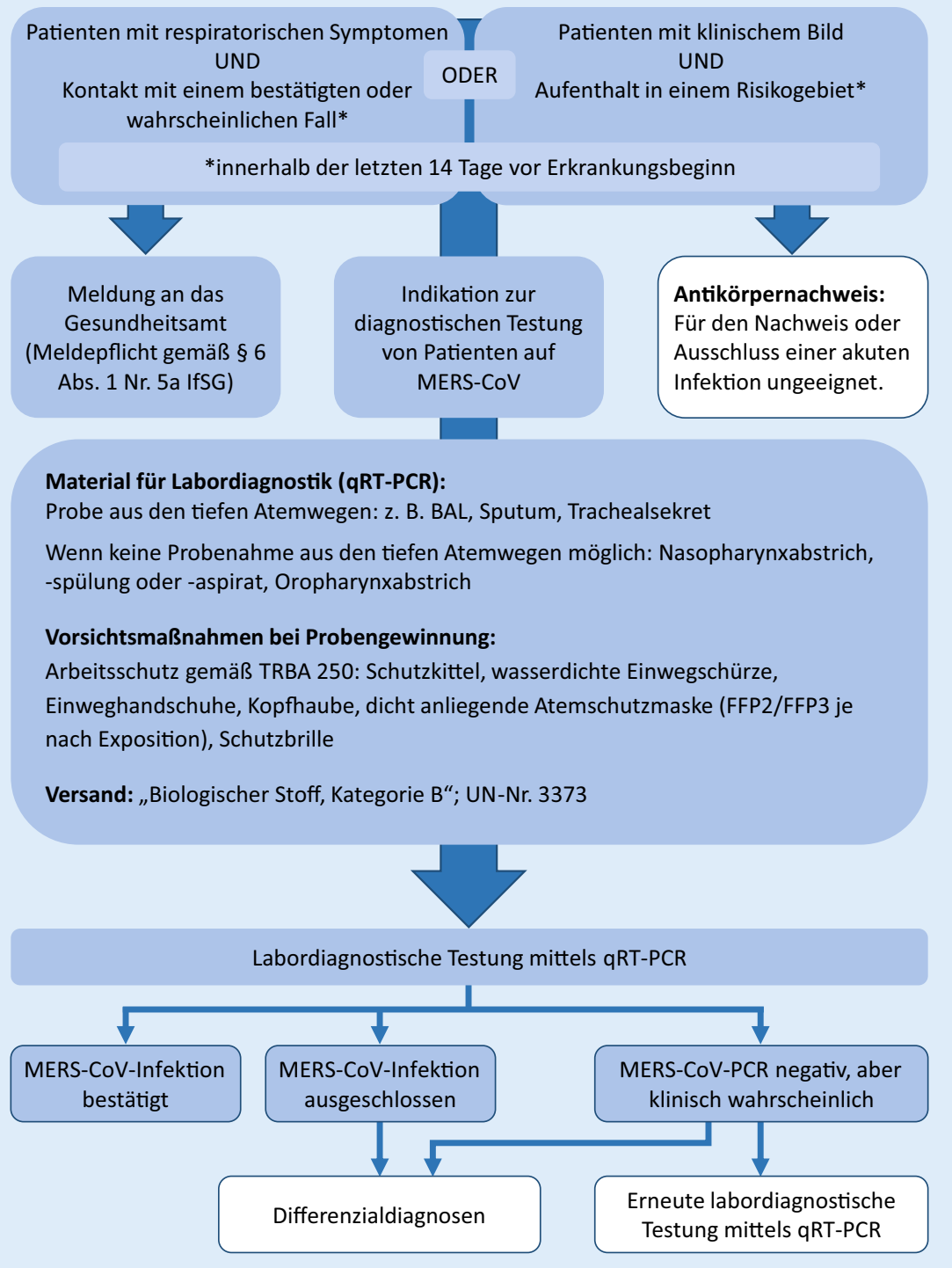

Abb. 3 \ Schema zur Einschätzung und Abklärung von MERS-Verdachtsfällen. BAL Bronchoalveoläre Lavage, FFP2/FFP3 Atemschutzklasse 2 oder 3 (,filtering face piece"), IfSG Infektionsschutzgesetz, MERS-CoV Middle-East-respiratory-syndrome-Coronaviren, $P C R$ Polymerase-Kettenreaktion, $q R T-P C R$ quantitative Echtzeit-Polymerase-Kettenreaktion, TRBA Technische Regeln für Biologische Arbeitsstoffe. (Adaptiert nach [22])

Die Umweltstabilität von CoV ist vergleichbar mit anderen behüllten RNAViren, die respiratorische Erkrankungen auslösen können, wie dem Influenzavirus. In Flüssigkeiten oder Sekreten aus dem Respirationstrakt bleiben $\mathrm{CoV}$ einige Tage infektiös, sind aber gegenüber Hitze, Detergenzien und Lösungsmitteln wie Ethanol relativ empfindlich.

Evolutionär betrachtet haben wahrscheinlich alle $\mathrm{CoV}$ ihr Reservoir in Nagetieren oder Fledermäusen, allerdings stellen diese Tiergruppen heutzutage für die vier im Menschen endemischen $\mathrm{CoV}$
Unabhängig von der Betrachtung der evolutionären Ursprünge gibt es keinen Hinweis darauf, dass auch aktuell die Virusvarianten aus Tieren auf den Menschen übergehen können. Somit ist die Infektionsquelle für alle vier endemischen $\mathrm{CoV}$ ausschließlich der Mensch. Im Gegensatz dazu besteht für die beiden zoonotischen CoV, SARS$\mathrm{CoV}$ und MERS-CoV, klare Evidenz für rezente Übergänge von Tieren auf den Menschen.

\section{MERS-Coronaviren}

Zoonotische Übergänge von MERS$\mathrm{CoV}$ zwischen Dromedaren und Menschen finden mindestens seit dem Jahr 2012 häufig statt. Dromedare stellen somit die Quelle für die primäre MERSCoV-Infektion des Menschen dar. Eine Übertragung auf den Menschen ist bei engem Kontakt mit den Tieren möglich. $\mathrm{Ob}$ das MERS-CoV nur durch direkten Kontakt zu Dromedaren oder auch über Tierprodukte übertragen werden kann, ist nicht eindeutig belegt. Da das Virus besonders im oberen Respirationstrakt von Dromedaren in sehr hohen Konzentrationen gefunden wird, ist die Übertragung durch Tröpfchen oder direkten Kontakt mit Sekreten am wahrscheinlichsten [14].

\section{》) MERS-CoV ist beim Menschen hauptsächlich in den tiefen Atemwegen zu finden}

MERS-CoV kann auch von einem Menschen auf einen anderen übertragen werden. Allerdings wurde eine kontinuierliche Übertragung in der Bevölkerung bislang nicht beobachtet; auch im normalen häuslichen Umgang finden nur wenige Übertragungen statt [6]. Diese Beobachtung steht im Gegensatz zu den beobachteten gravierenden krankenhausassoziierten Ausbrüchen auf der Arabischen Halbinsel und in Südkorea. Der scheinbare Widerspruch lässt sich dadurch erklären, dass MERS-CoV beim Menschen im Gegensatz zum Auftreten bei Kamelen hauptsächlich in den tiefen Atemwegen zu finden ist [4]. Im Rahmen der Behandlung eines infizierten Patienten, beispiels- 
weise mit Bronchoskopie, Beatmung und Absaugung, kann es zum Kontakt mit Sekreten aus den tiefen Atemwegen kommen, insbesondere wenn Infektionspräventionsmaßnahmen unzureichend umgesetzt werden.

\section{Hygiene und Prävention}

Aufgrund der wichtigen Rolle von nosokomialen Übertragungsketten sind Infektionspräventions- und Kontrollmaßnahmen sowie frühzeitige Diagnosestellungen entscheidend, um die Verbreitung von MERS-CoV einzudämmen. Bei engem Kontakt zu Patienten mit nachgewiesener MERS-CoV-Infektion oder Verdacht, insbesondere bei ausgeprägter Exposition gegenüber Sekreten aus der Lunge, etwa bei Intubation oder Bronchoskopie, sollten der Arbeitsschutz gemäß den Technischen Regeln für Biologische Arbeitsstoffe (TRBA) strikt eingehalten und geeignete Schutzmaßnahmen ergriffen werden (• Abb. $\mathbf{3}$ ).

Bei den bisher beschriebenen Fällen von MERS-CoV zeigte sich, dass die Diagnostik oft spät durchgeführt wurde, sodass es eine erhebliche Anzahl von exponierten Kontaktpersonen gab, in Deutschland beispielsweise bis zu 200. Für das Management solcher Kontaktpersonen von laborbestätigten symptomatischen MERS-Fällen gibt es Handlungsempfehlungen des RKI und des Europäischen Zentrums für die Prävention und die Kontrolle von Krankheiten (ECDC; [7, 21]). Dabei erfolgt grundsätzlich eine Unterteilung in Kontaktpersonen mit höherem Infektionsrisiko (Kategorie I) und geringerem Infektionsrisiko (Kategorie II): Beispielhafte Konstellationen für Kategorie I wären

- Personen mit mindestens 15-minütigem Gesichtskontakt,

- Personen mit direktem Kontakt zu infektiösen Körperflüssigkeiten und Sekreten,

- Personen mit Kontakt im Rahmen von Pflege oder medizinischer Untersuchung und auch

- Flugzeugpassagiere, die in derselben Reihe oder in den zwei Reihen vor oder hinter einem Patienten mit MERS gesessen haben.
Für alle Kontaktpersonen der Kategorie I wird eine namentliche Registrierung beim zuständigen Gesundheitsamt gefordert. Eine häusliche Absonderung und Isolation ist, solange keine Atemwegssymptomatik auftritt, nicht empfohlen, wohl aber eine Gesundheitsüberwachung bis zum 14. Tag nach dem letzten Kontakt. Zeigt eine Kontaktperson innerhalb dieser 14 Tage Symptome, die vereinbar mit einer MERS-CoV-Infektion sind, so gilt die Person als krankheitsverdächtig und sollte diagnostisch mittels RT-PCR abgeklärt werden. In diesem Fall ist die häusliche Isolation oder die Betreuung in einem Krankenhaus empfohlen. Bei Kontaktpersonen der Kategorie II werden keine routinemäßige Erfassung und Kontrolle bezüglich Krankheitssymptomen empfohlen.

Weitere aktualisierte und detaillierte Empfehlungen zum Management von MERS-CoV-Kontaktpersonen stehen über die Websites von RKI und ECDC zur Verfügung (https://www.rki.de und https://ecdc.europa.eu).
Um das Risiko von zoonotischen Übertragungen $\mathrm{zu}$ reduzieren, sollten Personen, die Kamelfarmen oder -märkte bzw. andere Orte besuchen, an denen Dromedare gehalten werden, allgemeine Hygienemaßnahmen einhalten und Kontakt mit Sekreten aus dem Respirationstrakt vermeiden. Kamelprodukte, die durch Kochen oder Pasteurisieren verarbeitet werden, sind für den Verzehr unbedenklich und stellen keine Infektionsquelle dar. Unbehandelte Kamelprodukte wie nichtpasteurisierte Milch sollten, obwohl es keinen gesicherten Hinweis auf Übertragung durch diese gibt, auch aus grundsätzlichen hygienischen Überlegungen gemieden werden.

\section{Therapie}

Für die Behandlung von CoV-Infektionen einschließlich SARS- und MERS$\mathrm{CoV}$ stehen keine spezifischen Medikamente oder Behandlungsregime zur Verfügung [27]. Die Gabe von
Hier steht eine Anzeige.

$$
\text { 包 Springer }
$$




\begin{tabular}{|l}
\hline Mehr Informationen zum Thema \\
\hline - Weltgesundheitsorganisation (WHO): \\
https://www.who.int/emergencies/mers- \\
cov/en// \\
- Robert Koch-Institut (RKI): https://www. \\
rki.de/DE/Content/InfAZ/M/MERS_ \\
Coronavirus/MERS-CoV.html \\
- Europäisches Zentrum für die Prävention \\
und die Kontrolle von Krankheiten (ECDC): \\
https://ecdc.europa.eu/en/middle-east- \\
respiratory-syndrome-coronavirus \\
Konsiliarlabor für Coronaviren: https:// \\
virologie-ccm.charite.de/diagnostik/ \\
konsiliarlaboratorium_fuer_coronaviren/
\end{tabular}

Interferon- $\alpha$ und Ribavirin reduzierte die (MERS-)CoV-Replikation in Zellkultur und in Tierversuchen. In einer Pilotstudie mit MERS-CoV-infizierten Patienten zeigte sich ein gering positiver Effekt. Aufgrund der in der Regel späten Diagnosestellung einer CoV-Infektion und der oft komplexen Situation, die im Rahmen einer intensivmedizinischen Behandlung auftreten kann, ist die Gabe im Einzelfall kritisch zu evaluieren $[16,19]$. Weitere therapeutische Optionen wurden vereinzelt eingesetzt, beispielsweise die Gabe von Rekonvaleszenzplasma, intravenösem Immunglobulin, monoklonalen Antikörpern oder Lopinavir/Ritonavir [1, 27].

Die Anwendung von Glukokortikoiden bei schwerer MERS-Erkrankung könnte mit einer erhöhten Mortalität einhergehen und sollte daher kritisch geprüft werden [27]. Trotz einer Vielzahl unterschiedlicher therapeutischer Ansätze gibt es weiterhin keine spezifischen therapeutischen Möglichkeiten, die bei der Behandlung von Patienten mit MERS-CoV überzeugen konnten. Entsprechend muss die Behandlung supportiv und abhängig vom klinischen Zustand des Patienten erfolgen.

Zur Prävention stehen allgemeine hygienische Maßnahmen und die frühe Diagnose mit strikter Isolation infizierter Patienten im Vordergrund. Die Isolation sollte bis zur wiederholten negativen Testung auf MERS-CoV in respiratorischem Sekret, in Anlehnung an das Vorgehen bei offener Lungentuberkulose und in Absprache mit der zuständigen Hygieneabteilung und dem öffentlichen Gesundheitsdienst erfolgen. Ebenso soll- ten Patienten mit Verdacht auf MERSCoV-Infektion isoliert werden, was in Deutschland insbesondere die Bedeutung einer detaillierten Reiseanamnese in den Vordergrund rückt.

\section{Impfung}

Ein Impfstoff steht nicht zur Verfügung, allerdings gibt es mehrere vielversprechende Kandidaten, die sich bereits in Tiermodellen (inklusive Kamel) als wirksam erwiesen haben. Erste klinische Studien wurden bereits initiiert. $\mathrm{Ob}$ eine Impfung angesichts der Seltenheit der Krankheit in relevantem Ausmaß Infektionen verhindern kann, ist Gegenstand aktueller Diskussionen. Allerdings könnte die Entwicklung eines Impfstoffs aufgrund der Omnipräsenz infizierter Kamele und der bereits identifizierten Risikogruppen - Krankenhausmitarbeiter in Risikogebieten und Personen mit engem Kontakt zu Kamelen - eine sinnvolle Strategie zur besseren Kontrolle von MERSCoV sein.

\section{Fazit für die Praxis}

- Vier der sechs bekannten humanpathogenen Coronaviren (CoV) sind endemisch und häufig. Sie rufen meist leichtere respiratorische Infektionen hervor. Schwere Verläufe sind selten und treten am ehesten bei Immunsupprimierten auf. Eine Erregerdiagnostik mittels EchtzeitReverse-Transkriptase-PolymeraseKettenreaktion (RT-PCR) an respiratorischem Material ist möglich, aber selten notwendig.

- Von den beiden epidemisch auftretenden CoV wurde das SARSCoV (SARS "severe acute respiratory syndrome") seit 15 Jahren weltweit nicht mehr nachgewiesen. MERS-CoV (MERS „Middle East respiratory syndrome“) ruft dagegen mit relevanter Häufigkeit schwere Pneumonien hervor.

- Der Verdacht auf eine MERS-Infektion sollte bei entsprechender Symptomatik und Reiseanamnese gestellt werden (Arabische Halbinsel, gegebenenfalls aktuelle Epidemiegebiete, enger Kontakt zu Kamelen oder Patienten).

- Die Isolierung des Patienten mit (vermuteter) MERS-CoV-Infektion und allgemeine Hygienemaßnahmen sind vordringlich.

- Der Nachweis von MERS-CoV erfolgt aus tiefem Atemwegsaspirat mittels RT-PCR. Die Probe muss unter besonderen Schutzmaßnahmen gewonnen und versandt werden. Eine Impfung oder spezifische Therapie ist bisher nicht etabliert.

\section{Korrespondenzadresse}

\section{Univ.-Prof. Dr. M. Witzenrath}

Arbeitsbereich Pulmonale Inflammation, Charité - Universitätsmedizin Berlin, corporate member of Freie Universität Berlin, HumboldtUniversität zu Berlin, and Berlin Institute of Health

Charitéplatz 1, 10117 Berlin, Deutschland martin.witzenrath@charite.de

\section{Einhaltung ethischer Richtlinien}

\section{Interessenkonflikt. V.M. Corman, J. Lienau und} M. Witzenrath geben an, dass kein Interessenkonflikt besteht.

Für diesen Beitrag wurden von den Autoren keine Studien an Menschen oder Tieren durchgeführt. Für die aufgeführten Studien gelten die jeweils dort angegebenen ethischen Richtlinien.

\section{Literatur}

1. Arabi YM, Alothman A, Balkhy HH et al (2018) Treatment of middle east respiratory syndrome with a combination of lopinavir-ritonavir and interferon-beta1b (MIRACLE trial): study protocol for a randomized controlled trial. Trials 19:81

2. Chu DKW, Hui KPY, Perera R et al (2018) MERS coronaviruses from camels in Africa exhibit regiondependent genetic diversity. Proc Natl Acad Sci USA 115:3144-3149

3. Corman VM, Muller MA, Costabel U et al (2012) Assays for laboratory confirmation of novel human coronavirus (hCoV-EMC) infections. Euro Surveill. https://doi.org/10.2807/ese.17.49.20334-en

4. Corman VM, Albarrak AM, Omrani AS et al (2016) Viral shedding and antibody response in 37 patients with middle east respiratory syndrome Coronavirus infection. Clin Infect Dis 62:477-483

5. Corman VM, Muth D, Niemeyer D et al (2018) Hosts and sources of endemichuman Coronaviruses. Adv Virus Res 100:163-188

6. Drosten C, Meyer B, Muller MA et al (2014) Transmission of MERS-coronavirus in household contacts. N Engl J Med 371:828-835

7. Ecdc (2014) European Centre for Disease Prevention and Control. Severe respiratory disease 
associated with Middle East respiratory syndrome coronavirus (MERS-CoV) - tenth update, 31 May 2014. European Centre for Disease Prevention and Control, Stockholm

8. Eichenberger EM, Soave R, Zappetti D et al (2018) Incidence, significance, and persistence of human coronavirus infection in hematopoietic stem cell transplant recipients. Bone Marrow Transplant. https://doi.org/10.1038/s41409-018-0386-z

9. Esper F, Ou Z, Huang YT (2010) Human coronaviruses are uncommon in patients with gastrointestinal illness. J Clin Virol 48:131-133

10. Friedman N, Alter H, Hindiyeh Metal (2018) Human Coronavirus infections in Israel: epidemiology, clinical symptoms and summer seasonality of HcoV-HKU1. Viruses. https://doi.org/10.3390/ v10100515

11. Hui DS, Azhar El, Kim YJ et al (2018) Middle East respiratory syndrome coronavirus: risk factors and determinants of primary, household, and nosocomial transmission. Lancet Infect Dis 18:e217-e227

12. International Committee on Taxonomy of Viruses (2019) ICTV Virus Taxonomy: 2018b Release

13. Kiambi S, Corman VM, Sitawa R et al (2018) Detection of distinct MERS-Coronavirus strains in dromedary camels from Kenya, 2017. Emerg Microbes Infect 7:195

14. Mackay IM, Arden KE (2015) MERS coronavirus: diagnostics, epidemiology and transmission. Virol J 12:222

15. Mackay IM, Arden KE, Speicher DJ et al (2012) Cocirculation of four human coronaviruses ( $\mathrm{HCoVs}$ ) in Queensland children with acute respiratory tract illnesses in 2004. Viruses 4:637-653

16. Mayer K, Nellessen C, Hahn-Ast C et al (2016) Fata outcome of human coronavirus NL63 infection despite successful viral elimination by IFN-alpha in a patient with newly diagnosed ALL. Eur J Haematol 97:208-210

17. Mehand MS, Al-Shorbaji F, Millett $P$ et al (2018) The WHO R\&D Blueprint: 2018 review of emerging infectious diseases requiring urgent research and development efforts. Antiviral Res 159:63-67

18. Morfopoulou S, Brown JR, Davies EG et al (2016) Human Coronavirus OC43 associated with fatal encephalitis. N Engl J Med 375:497-498

19. Omrani AS, Saad MM, Baig K et al (2014) Ribavirin and interferon alfa-2a for severe Middle East respiratory syndrome coronavirus infection: a retrospective cohort study. Lancet Infect Dis 14:1090-1095

20. Paloniemi M, Lappalainen S, Vesikari T (2015) Commonly circulating human coronaviruses do not have a significant role in the etiology of gastrointestinal infections in hospitalized children JClin Virol 62:114-117

21. RKI (2014) Empfehlungen des RKI für das Management von Kontaktpersonen laborbestätigter symptomatischer MERS-Fälle; Stand 3. Juli 2014. In, Berlin https://www.rki.de/DE/Content/InfAZ/ M/MERS_Coronavirus/MERS-CoV_Management_ Kontaktpersonen.html

22. RKI (2015) Schwere respiratorische Erkrankungen in Verbindung mit Middle East Respiratory Syndrome Coronavirus (MERS-CoV). FALLDEFINITION zur Fallfindung, Meldung und Übermittlung. https://www.rki.de/DE/Content/InfAZ/M/ MERS_Coronavirus/Corona_Falldefinition.pdf? blob=publicationFile. Zugegriffen: 1. Aug. 2019

23. Shaman J, Morita H, Birger R et al (2018) Asymptomatic summertime shedding of respiratory viruses. J Infect Dis 217:1074-1077
24. Van Elden LJ, Van Loon AM, Van Alphen F et al (2004) Frequent detection of human coronaviruses in clinical specimen from patients with respiratory tract infection by use of a novel real-time reversetranscriptase polymerase chain reaction. J Infect Dis 189:652-657

25. WHO (2004) Severe acute respiratory syndrome (SARS) - Disease outbreak news, May 2004. In: https://www.who.int/csr/sars/archive/en/

26. WHO (2018) Laboratory testing for middle east respiratory syndrome Coronavirus, interim guidance (WHO/MERS/LAB/15.1/rev1/2018). World Health Organization, Geneva, Switzerland

27. WHO (2019) Clinical management of severe acute respiratory infection when Middle East respiratory syndrome coronavirus (MERS-CoV) infection is suspected. Interim guidance, Updated January 2019 (WHO/MERS/Clinical/15.1 Revision 1). World Health Organization, Geneva, Switzerland

28. WHO (2019) Middle East respiratory syndrome coronavirus (MERS-CoV) - MERS situation update, April 2019. In: https://www.who.int/emergencies/ mers-cov/en/

29. Yeh EA, Collins A, Cohen ME et al (2004) Detection of coronavirus in the central nervous system of a child with acute disseminated encephalomyelitis. Pediatr Electron Pages 113:e73-76

30. Zaki AM, Van Boheemen S, Bestebroer TM et al (2012) Isolation of a novel coronavirus from a man with pneumonia in Saudi Arabia. N Engl J Med 367:1814-1820

31. Zohaib A, Saqib M, Athar MA et al (2018) Countrywide survey for MERS-Coronavirus antibodies in dromedaries and humans in Pakistan. Virol Sin 33:410-417

\section{Lungenembolie}

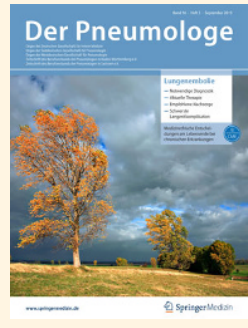

Die akute Lungenembolie ist eine häufige Erkrankung, die mit einer hohen Mortalität und Morbidität einhergeht. Die Inzidenz liegt nach Autopsiestudien und epidemiologischen Schätzungen bei etwa 1-2 / 1000 Menschen pro Jahr und steigt altersabhängig. Bei einem Teil der Betroffenen verläuft eine Lungenembolie fast asymptomatisch und wird nur als Zufallsbefund diagnostiziert.

Mit den Artikeln des Leitthemas in Der Pneumologe 05/2019 erhalten Sie einen Überblick über die Notwendigkeiten im Umgang mit der akuten Lungenembolie sowie Empfehlungen für die Therapie und Nachsorge.

- Diagnostik der akuten Lungenembolie - was ist notwendig, was ist entbehrlich?

- Aktuelle Therapie der akuten Lungenembolie

- Nachsorge bei Lungenembolie - was ist empfehlenswert?

- Chronisch thromboembolische pulmonale Hypertonie - aktuelle Diagnostik und Therapie

Suchen Sie noch mehr zum Thema?

Mit e.Med - den maßgeschneiderten Fortbildungsabos von Springer Medizin - haben Sie Zugriff auf alle Inhalte von SpringerMedizin.de. Sie können schnell und komfortabel in den für Sie relevanten Zeitschriften recherchieren und auf alle Inhalte im Volltext zugreifen.

Weitere Infos zu e.Med finden Sie auf springermedizin.de unter "Abos" 